\section{Total adenosine deaminase highly correlated with adenosine deaminase 2 activity in serum}

We read with great interest the article published by Lee and colleagues ${ }^{1}$ presenting the potential diagnostic value of adenosine deaminase 2 (ADA2) in systemic juvenile idiopathic arthritis (sJIA) with macrophage activation syndrome (MAS). Their study examined ADA2 activity in children with inflammatory and immune-mediated diseases. By comparing normal ranges of $\mathrm{ADA} 2$ in healthy individuals, their finding identified the utility of plasma ADA2 activity as a biomarker of MAS. ADA2 activity were measures by a spectrophotometric assay in the presence of a selective inhibitor of ADA1, erytro-9-amino$\beta$-hexyl- $\alpha$-methyl-9-ethanol hydrochloride (EHNA). We support the views expressed by the authors that ADA2 detection could be helpful in the diagnosis of MAS in sJIA. Here, we would like to share with the authors our data of ADA activity detection.

As we know, ADA contains two isoenzymes: ADA1 an ADA2. In plasma, $\mathrm{ADA} 2$ is a major component of total adenosine deaminase (tADA). ${ }^{2}$ tADA activity detection has been carried out in the clinical laboratory for many years, which was usually used for differential diagnosis of benign and malignant effusions. ${ }^{4}$ The measurement method of tADA activity is simpler than ADA2 activity. Here, we report the correlation between tADA and ADA2 activity in the serum of healthy individuals and patients with immune-mediated diseases. Serum tADA activity was measured with an enzymatic method kit (Sichuan Maccura Biotechnology, China), adapted to the automated biochemistry analyser (Hitachi 7600, Japan). ${ }^{5}$ Serum ADA2 activity was measured in the presence of $0.1 \mathrm{mM}$ EHNA. We determined serum tADA and ADA2 activity in 386 healthy individuals and 430 patients with different immune-mediated diseases, including rheumatoid arthritis, systemic lupus erythematosus, ankylosing spondylitis, myasthenia gravis and autoimmune liver diseases.

First, we found that tADA levels highly correlated with ADA2 in 816 individuals (figure $1 \mathrm{~A} ; \mathrm{r}=0.921, \mathrm{p}<0.0001$ ). Notably, the correlation coefficient between tADA and ADA2 in patients with immune-mediated diseases (figure $1 B ; r=0.947, p<0.0001$ ) was higher than that in healthy individuals (figure $1 \mathrm{C} ; \mathrm{r}=0.860$, $\mathrm{p}<0.0001)$. Thus, like ADA2 activity, tADA activity might also be
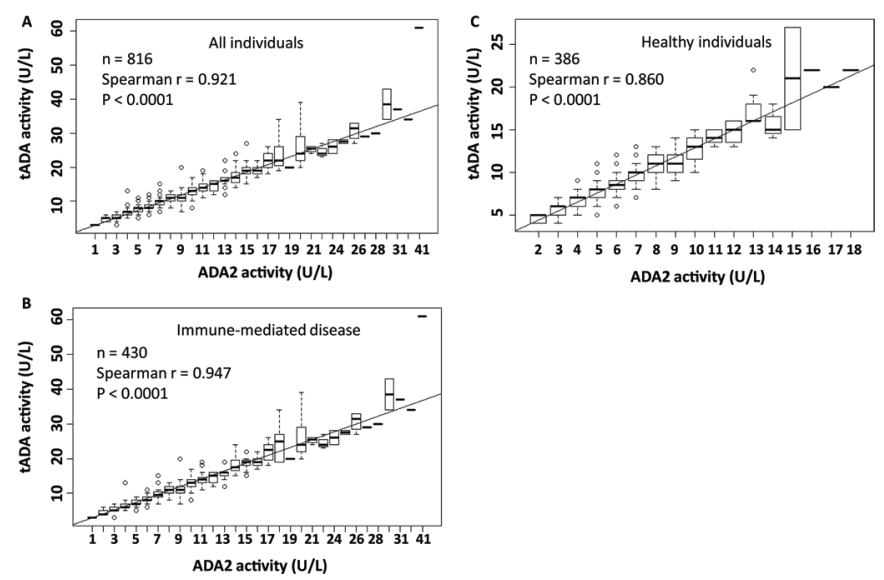

Figure 1 Correlation between serum ADA2 and TadA activity was calculated by Spearman's rank correlation analysis. (A-C) Correlation between ADA2 and TadA in all 816 individuals, 430 patients with immune-mediated diseases and 385 healthy individuals. ADA2, adenosine deaminase 2; TadA, total adenosine deaminase.
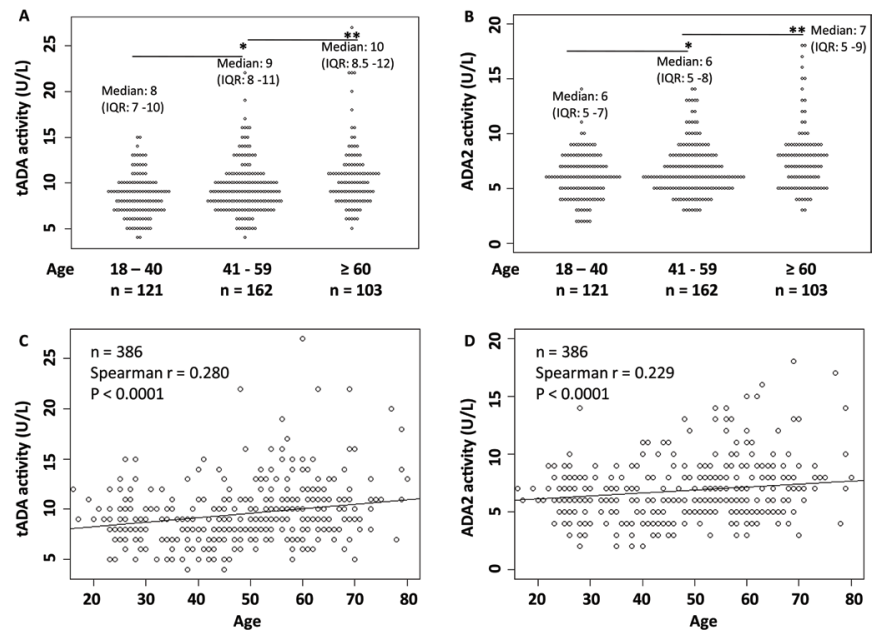

Figure 2 Determination of serum TadA and ADA2 activity in healthy adults. $(A, B)$ Plot comparing serum TadA and ADA2 activity in adults stratified by age. $(C, D)$ Correlation between serum TadA, $A D A 2$ activity and age. ${ }^{*} p<0.05,{ }^{* *} p<0.01$. ADA2, adenosine deaminase 2 ; TadA, total adenosine deaminase.

a biomarker of MAS. Due to convenient detection, tADA activity would be more suitable for clinical application than ADA2.

Second, in the study of Lee et al, ${ }^{1}$ they showed that ADA2 activity was higher in children than in adults (age 18 years and older), with an overall negative correlation with age $(\mathrm{r}=-0.250, \mathrm{p}<0.0001)$. Here, we analysed the distribution of tADA and ADA2 activity in adults. The results showed that tADA and ADA2 activity were higher in elderly people (age $\geq 60$ years) than young adults (figure $2 \mathrm{~A}, \mathrm{~B} ; \mathrm{p}<0.01$ ), with an overall positive correlation with age (figure $2 \mathrm{C}, \mathrm{D} ; \mathrm{p}<0.0001$ ). Because ADA plays an important role in the immune system, these combined data indicated the potential difference of the immune system status between children, elderly people and other adults.

In conclusion, the high correlation between tADA and ADA2 activity supports the clinical application value of tADA detection. Further studies are needed to validate the effect and mechanism of higher level of ADA activity in children and elderly people.

\section{Zhao-Wei Gao • , Xi Wang, Fang Lin, Ke Dong}

Department of Clinical Laboratories, Fourth Military Medical University, Xi'an, China

Correspondence to Dr Fang Lin and Dr Ke Dong, Department of Clinical Diagnosis, Tangdu Hospital, Air Force Medical University, Xinsi Road, Xi'an, Shanxi 710038, China; linfang168@hotmail.com, tdjyk3@fmmu.edu.cn

Contributors Z-WG and KD performed the study design. Z-WG and FL analysed the data and wrote the manuscript. XW performed the experiments. All authors approved the final manuscript.

Funding This work was supported by grants from the National Natural Science Foundation of China (grant number 81702732).

Competing interests None declared.

Patient consent for publication Not required.

Provenance and peer review Not commissioned; internally peer reviewed.

(c) Author(s) (or their employer(s)) 2020. No commercial re-use. See rights and permissions. Published by BMJ.

\section{Check for updates}

To cite Gao Z-W, Wang X, Lin F, et al. Ann Rheum Dis Epub ahead of print: [please include Day Month Year]. doi:10.1136/annrheumdis-2020-217007

Received 17 January 2020

Accepted 20 January 2020 
Ann Rheum Dis 2020;0:1-2. doi:10.1136/annrheumdis-2020-217007

ORCID iD

Zhao-Wei Gao http://orcid.org/0000-0003-4477-0966

\section{REFERENCES}

1 Lee PY, Schulert GS, Canna SW, et al. Adenosine deaminase 2 as a biomarker of macrophage activation syndrome in systemic juvenile idiopathic arthritis. Ann Rheum Dis 2019. doi:10.1136/annrheumdis-2019-216030. [Epub ahead of print: 09 Nov 2019].
2 Ratech H, Martiniuk F, Borer WZ, et al. Differential expression of adenosine deaminase isozymes in acute leukemia. Blood 1988;72:1627-32.

3 Iwaki-Egawa S, Yamamoto T, Watanabe Y. Human plasma adenosine deaminase 2 is secreted by activated monocytes. Biol Chem 2006;387:319-21.

4 Koh KK, Kim EJ, Cho CH, et al. Adenosine deaminase and carcinoembryonic antigen in pericardial effusion diagnosis, especially in suspected tuberculous pericarditis Circulation 1994:89:2728-35

5 Gao Z-W, Zhao G-H, Zhang Z, et al. Serum adenosine deaminase activity is increased in systemic lupus erythematosus patients and correlated with disease activity. Immunol Res 2018:66:299-304 